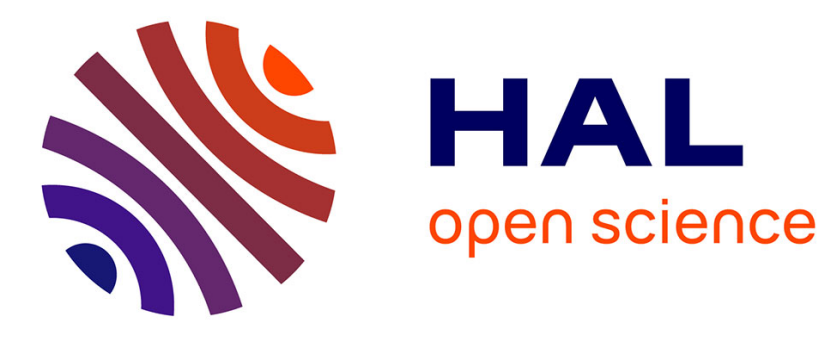

\title{
Jet instability of a shear-thickening concentrated suspension
}

Maxime Liard, Akihiro Sato, Jérémy Sautel, Didier Lootens, Pascal Hébraud

\section{To cite this version:}

Maxime Liard, Akihiro Sato, Jérémy Sautel, Didier Lootens, Pascal Hébraud. Jet instability of a shear-thickening concentrated suspension. European Physical Journal E: Soft matter and biological physics, 2020, 43 (11), 10.1140/epje/i2020-11994-3 . hal-03093091

\section{HAL Id: hal-03093091 \\ https://cnrs.hal.science/hal-03093091}

Submitted on 3 Jan 2021

HAL is a multi-disciplinary open access archive for the deposit and dissemination of scientific research documents, whether they are published or not. The documents may come from teaching and research institutions in France or abroad, or from public or private research centers.
L'archive ouverte pluridisciplinaire HAL, est destinée au dépôt et à la diffusion de documents scientifiques de niveau recherche, publiés ou non, émanant des établissements d'enseignement et de recherche français ou étrangers, des laboratoires publics ou privés. 


\title{
Jet instability of a shear-thickening concentrated suspension
}

\author{
Maxime Liard ${ }^{1,2}$, Akihiro Sato ${ }^{1}$, Jérémy Sautel ${ }^{1}$, Didier Lootens ${ }^{2}$, and Pascal Hébraud ${ }^{1}$ \\ 1 Université de Strasbourg, IPCMS/CNRS UMR 7504, 23 rue du Loess, 67034 Strasbourg, FRANCE \\ 2 Sika Technology AG, Tuffenwies 16, 8048 Zürich, SWITZERLAND
}

Received: date / Revised version: date

\begin{abstract}
We investigate the flow of a concentrated suspension of colloidal particles at deformation rates higher than the discontinuous shear thickening transition shear rate. We show that, under its own weight, a jet of a concentrated enough colloidal suspension, simultaneously flows while it sustains tensile stress and transmits transverse waves. This results in a new flow instability of jets of shear-thickening suspensions: the jet is submitted to rapid transverse oscillations, that we characterize.
\end{abstract}

PACS. PACS-key 82.70.Dd - PACS-key 83.60.Rs

\section{Introduction}

Submitted to high strain rates, the flow of concentrated suspensions exhibits a transition towards a strongly shear thickening behavior [1-3], both in the colloidal and in the non-colloidal regimes [4]. The deformation rate and the volume fraction at which shear thickening develops depend on the exact nature of the suspension, in particular on the shape and smoothness of the particles $[5,6]$ and their short distance interactions [7], being lubrication [8] or friction interactions [9].

Under high shear rates, the suspension strongly shear thickens; at higher shear stresses, the suspension is able to sustain a constant applied stress : it is jammed [11]. Both states have a finite lifetime after the cessation of shear [12]. These behaviors may be rationalized by assuming that a fabric of force chain develops under shear [10] and, that, above some shear rate, becomes dense enough to yield a jammed state [11]. A phenomenological model has been proposed for non-Brownian suspensions [13] in which shearthickening appears as a first order transition between two Newtonian branches of flow: a lubricated one whose viscosity diverges at some volume fraction and a frictional one whose viscosity diverges at a lower volume fraction. Assuming that particles can make contacts, a stress scale appears above which the lubricated branch of flow is unstable. Depending on the volume fraction of the suspension, the transition leads to a discontinuity in the apparent viscosity, which defines the discontinuous shear transition (DST). This describes well the experimentally measured phase diagram [11].

At the discontinuous shear thickening transition (DST), the suspension becomes dilatant: a strong normal force develops at or close to the transition. Dilatance is related to the existence of a compression axis under the applied deformation and to the formation of a force network $[11,14]$.
One may thus expect that the nature of the deformation flow plays a role in the DST. Experiments in extensional geometry using corn starch suspensions, demonstrate that shear DST occurs at extension rates one order of magnitude smaller than the corresponding shear rate in a simple shear flow [15]. The absence of a rotational component of the deformation field thus seems to promote the shear thickening behavior. On the opposite, it has been observed [16] by Stokesian Dynamics simulations of the flow of dense suspensions that shear thickening occurs at similar deformation rates under a simple shear and an extensional shear flow. Moreover, the confinement of the particles, either by solid boundaries or by surface tension is usually thought to be necessary to maintain the jammed state [17] and indeed, free surfaces become rough at the jamming transition due to the particles pressure. Nevertheless, jamming also occurs far from boundaries: it appears as a transient response to an impact to the suspension and a solid-like region is separated from a fluid zone by a solidification front $[18,19]$.

Most of the previous observations have been performed under shear, using geometries in which a stress is applied at the surface.

Here, we wish to study the response of a concentrated suspension to the application of a controlled stress in a geometry where the suspension is free to dilate in all directions. Contrasting to extension experiments performed at controlled extension rate, where one pulls at both extremities of a column of material, we study the emptying of a funnel. The falling extremity of the column of liquid is free, and the end-to-end distance is not fixed by external conditions. This is a relevant situation in many natural flows, such as geophysical mud flows [20] and industrial processes that imply extrusion [21]. We perform an extrusion experiment in which the stress is imposed by gravity. We are not interested in the flow inside a pipe where it 
has been shown that particles may jam under the application of an external pressure [22], but by the flow at the outlet. The dynamics and stability of a Newtonian jet are well understood $[23,24]$, but we consider here a strongly non-Newtonian behavior. We show that, under these conditions, DST occurs, leading to a transverse instability.

\section{Experimental setup}

We consider a suspension of silica particles (FOC, New Bedford, USA), dispersed in water at well controlled $\mathrm{pH}=7$. Their radius is $775 \pm 75 \mathrm{~nm}$, as measured by dynamic light scattering. A suspension at $\phi=0.5$ is first prepared. Then, using this batch as a reference, and working in a humidity saturated glove box in order to avoid evaporation, the studied samples are prepared by dilution or by the addition of dry silica powder to a volume fraction ranging from 0.48 to 0.54 . At the highest volume fraction, DST occurs at such low deformation rates that the suspension is difficult to handle. $10 \mathrm{~mL}$ of the suspension are placed in a funnel made of a polyacrylate resin. The funnel angle is $\alpha=30^{\circ}$ and its output diameter $2 a=3 \mathrm{~mm}$. We measure the evolution of the weight of the funnel as a function of time when the suspension falls down as a gravitational jet. The jet is imaged at $500 \mathrm{im} / \mathrm{s}$ (Mikrotron EOS CL). The preparation of the suspension and all of the experiments are performed in a glove box at humidity ratio $\approx 97 \%$ in order to avoid evaporation. By weighing a suspension over several hours, much longer than a typical jet experiment, we checked that evaporation is smaller than $10^{-5} \mathrm{w} / \mathrm{w}$. $\min ^{-1}$, which is negligible during the duration of the experiment (less than $10 \mathrm{~min}$ ).

\section{Rheological behaviour of the suspensions}

The rheological properties of the suspensions at different volume fractions have been measured as a function of the shear rate, in a cone/plate geometry (Anton Paar MCR301, angle $1^{\circ}$, radius $25 \mathrm{~mm}$ and truncation gap $50 \mu \mathrm{m})$ at controlled shear rate between 1 and up to 1000 $\mathrm{s}^{-1}$. The normal and tangential stresses are measured for $30 \mathrm{~s}$ at each shear rate value. The results are plotted in Fig. 1. At all the considered volume fractions, the suspensions shear thin at low shear rates, and viscosity fluctuations, precursors to the shear-thickening transition, may be observed [25].Then, at higher shear rates, they shear thicken. The onset of shear thickening decreases with the volume fraction. Then, for $\phi \geq 0.53$, the suspension exhibits DST, and shear thickening is associated to a divergence of the normal stress (Fig. 1 top). These behaviors are characteristic of many of the systems for which discontinuous shear thickening and shear jamming have been reported $[6,25]$, although the values of the volume fraction and the shear rate at which jamming occurs depend on the interactions between particles $[1,6,26]$.

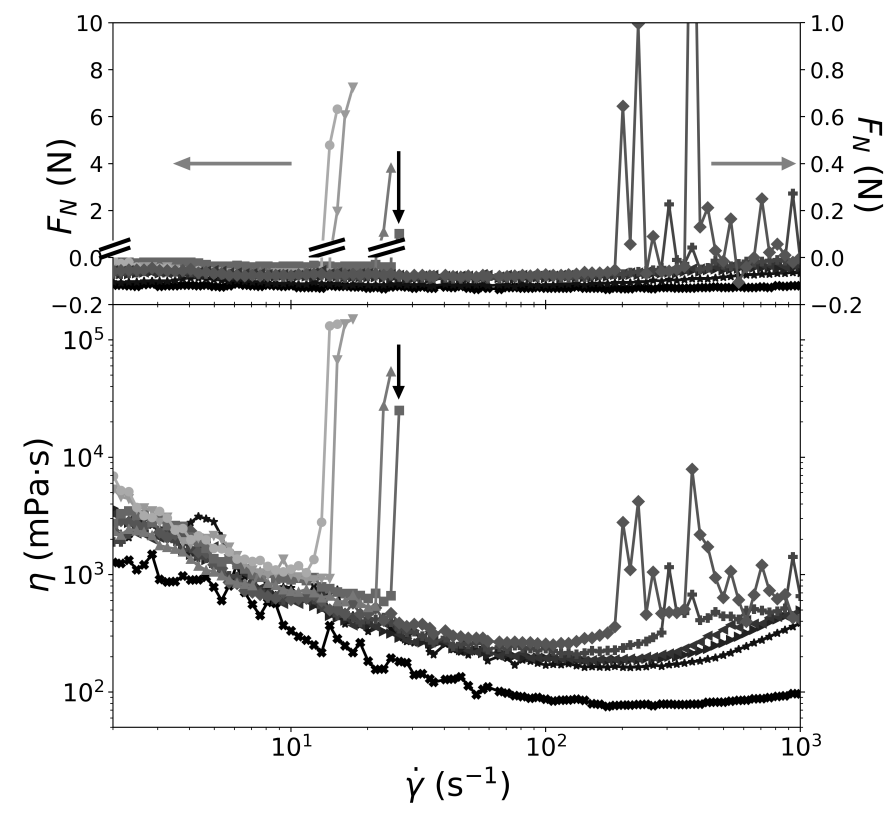

Fig. 1. Bottom : Evolution of the viscosity as a function of the shear rate $\dot{\gamma}$, for $\phi=0.48(\times), 0.5(\star), 0.505(\bullet), 0.51(\triangleleft)$, $0.515(+), 0.52(\bullet), 0.53(\boldsymbol{\square}), 0.5325(\boldsymbol{\Delta}), 0.5375(\boldsymbol{\nabla}), 0.54(\bullet)$, from black to light grey. Top : evolution of the normal force as a function of the shear rate for the same suspensions. Data corresponding to volume fractions $\phi=0.48$ to 0.52 refer to the right axis coordinate. Out of range point corresponds to $\phi=$ 0.52 and has coordinates $\left(376 \mathrm{~s}^{-1}, 2.165 \mathrm{~N}\right)$. Data for volume fraction 0.53 to 0.54 refer to the left axis coordinate (the axis is broken at 0 and its scale is smaller for positive values). The vertical black arrows point to the curve corresponding to $\phi=$ 0.53 .

\section{Jet flows}

\section{Low volume fraction}

Let us now consider the free fall of a jet of such a suspension. Several flow regimes are observed, as a function of the volume fraction. At low volume fraction $(\phi<49 \%$, Fig. 2(a)), the shape of the jet cannot be distinguished from the free fall of a Newtonian fluid. From the evolution of the mass with time, the volume flow may be computed at the output and the extensional rate at the output of the funnel, $\dot{\epsilon}$ may be evaluated. Considering the radius $a$ of the funnel as a characteristic length and computing the velocity $U$ of the fluid from the time needed for $10 \mathrm{~mL}$ of suspension to flow out of the funnel of output radius $a$, relevant adimensional flow numbers are computed. Reynolds number $R e=\frac{\rho U a}{\eta}, \rho$ being the density of the suspension and $\eta$ its viscosity at $10 \mathrm{~s}^{-1}$, measured in Fig. 1, compares the inertia to viscous forces. Weber number, $W e=\frac{\rho U^{2} a}{\sigma}$, $\sigma=72 \cdot 10^{-3} \mathrm{~N} \cdot \mathrm{m}^{-1}$ being the surface tension, measured using the pendant droplet technique (Teclis tensiometer) and that does not depend on the silica particles concen- 
tration, is the ratio of the kinetic energy of a droplet coming from the jet and to its surface energy. Lastly, Froude number, $\mathrm{Fr}=\frac{U^{2}}{g a}, g$ being the acceleration of gravity, compares gravitational to inertia forces. At low volume fractions, all these adimensional numbers are much larger than 1 (Fig. 2(e)) and the flow is inertial. In this regime, the shape of the jet is solely given by the conservation of energy and conservation of mass: the shape of the jet can be described ignoring viscous losses. Writing Bernoulli's law and mass conservation, one finds the radius of the jet $r$ as a function of the distance from the funnel output, $z[23]$ :

$$
\begin{aligned}
r(z) & =a\left(1+\frac{2}{\operatorname{Fr}} \frac{z}{a}+\frac{2}{W e}\left(1-\frac{a}{r}\right)\right)^{-1 / 4} \\
& \approx a\left(1+\frac{2}{\operatorname{Fr}} \frac{z}{a}\right)^{-1 / 4}
\end{aligned}
$$

where we have taken into account that the last term of the sum, involving the Weber number, is approximately one tenth of the first two terms.
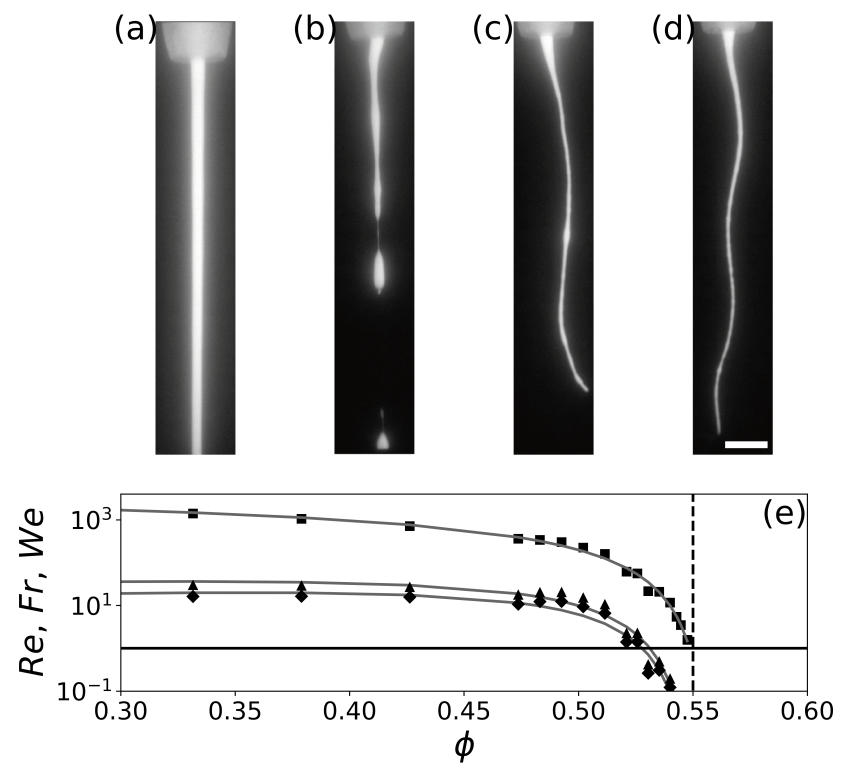

Fig. 2. (a) to (d) Images of the falling jet at $\phi=0.48,0.515$, 0.5325 and 0.5375 out of a funnel of output diameter $3 \mathrm{~mm}$. White bar $=1 \mathrm{~cm}$. (e) Reynolds (ם), Froude $(\mathbf{\Lambda})$ and Weber ( ) numbers at the output of the funnel, as a function of the volume fraction. Grey lines are guides to the eye. The solid line marks a value of 1 .

This describes very well the shape of the jet with no adjustment parameter (Fig. 3 (a) light grey curve).

\section{Intermediate volume fraction}

As the volume fraction of the jet increases, the viscosity of the suspension increases and the jet velocity decreases, so that the role of surface tension becomes dominant. The Weber number being of the order of 1 , interfacial instabilities advected by the jet develop: their velocity does not depend on the volume fraction. The amplitude of capillary waves increases exponentially from the output of the funnel, with a characteristic length $\ell=\sqrt{W e} f(k R)$ where $k$ is the wave number of the considered wave and $R$ the radius of the jet $[27,28]$. In the range of concentration where droplets are observed, both the radius of the jet and the wave number of the dominant mode are approximately constant, so that $\frac{\ell}{2 a}=\alpha \sqrt{W e}$ where $\alpha$ is a dimensional number, independent of the system and that should be determined experimentally [27]. The evolution of the length of the jet with $\sqrt{W e}$ is plotted in Fig. 3(c). We indeed obtain a linear relationship between $\ell$ and $\sqrt{W e}$ and $\alpha=9.1$, slightly smaller than the coefficient obtained by Weber $(\alpha=12)$. Nevertheless, it has been argued [29] that this coefficient might not be constant and may depend on the flow rate, which may explain the observed discrepancy.

\section{High volume fraction}

But one observes a transition to another regime : at $\phi=$ 0.53 , the behavior of the jet dramatically changes. Its length suddenly increases (Fig. 3(c) and (d)) and one no longer observes the occurence of droplets. The jet breaks before reaching the solid surface under the funnel and solid-like chunks of suspension fall down onto the surface. They keep a cylindrical shape for a few seconds before they become spherical, after a few seconds, in agreement with previously measured finite time of the jammed state [12]. The shape of the jet is markedly different in this regime. Its radius $r(z)$ is no longer described by Eq. 2. As will be discussed later, the jet does not remain vertical, but exhibits oscillations (Fig. 2 (d)). The curvilinear distance along the jet is thus different from the height and we now compute its radius at a given vertical height, $z$. Fig. 3(a) gives its shape as a function of $z$. Two regimes may be distinguished: close to the output of the funnel, the jet radius decreases linearly, defining a conical shape of angle $\sim 80^{\circ}$ that does not depend on $\phi$ in the narrow volume fraction window where it is defined (between 0.53 and 0.54 ). Then, the diameter of the jet is slightly decreasing.

It may be expected that, at some position of the jet, the deformation rate reaches the critical rate at which the suspensions shear thickens. Let us compute the local extension along the jet, $\epsilon_{l}(z)=\partial h / \partial z$, where $h$ is the thickness of a slice along the jet. Writing the volume conservation along the jet, $\epsilon_{l}(z)=-2 \partial R / \partial z h(z) / R(z), R(z)$ being the radius of the jet at position $z$, leading to the local extensional rate :

$$
\dot{\epsilon}_{l}=\epsilon \frac{Q}{\pi R(z)^{2} h(z)}=-\frac{2 Q}{\pi R(z)^{3}} \frac{\partial R}{\partial z}
$$



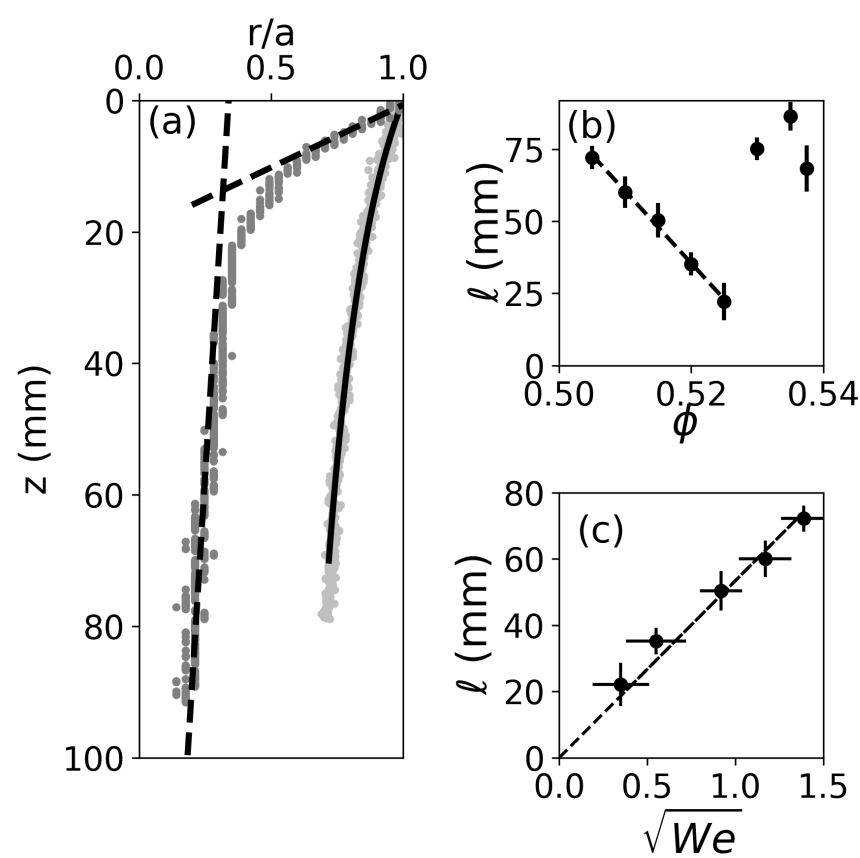

Fig. 3. (a) Shape of the jet at $\phi=0.49$ (light grey points) and $\phi=0.5325$ (dark points). Continuous curve is a fit of the shape of the jet in the fluid regime according to the shape of a Bernoulli jet, $r(z)=a\left(1+\frac{2}{F r} \frac{z}{a}\right)^{-1 / 4}$ with no adjustment parameter. Dashed lines are fits of the shape of the jet in the jammed regime at the output of the funnel (up to $8 \mathrm{~mm}$ below) and far from the output (40 to $80 \mathrm{~mm}$ below the output). (b) Evolution of the average length (calculated from the opening of the funnel output until the emptying of the funnel), $\ell$, as a function of the volume fraction. Dashed lines are guides to the eye. (c) Length of the jet as a function of $\sqrt{W e}$ in the droplet regime. Dashed line is a linear fit of the data, leading to $: \ell=54.6 \sqrt{W e} \mathrm{~mm}$. Error bars represent one standard deviation of $\ell$, obtained by repeating the measurement 3 to 7 times.

where $Q$ is the volume flow rate, computed from the evolution of mass of the jet with time. The extensional deformation rate is plotted as a function of the distance from the output of the funnel in Fig. 4 inset. It first increases at the output of the funnel, in the conical region of the jet, and reaches a maximum, close to the transition to an almost constant radius jet. The position of the maximum extension rate along the jet is indicated by horizontal segments in Fig. 4, main graph. The value of the maximum extensional rate increases when the output funnel diameter increases, from $14 \mathrm{~s}^{-1}$ (output diameter $3 \mathrm{~mm}$ ), to $65 \mathrm{~s}^{-1}$ (diameter $7 \mathrm{~mm}$ ). These values are of the same order of magnitude than the critical strain rate measured by rheology (Fig. 1, $\dot{\gamma}_{c}=23 \mathrm{~s}^{-1}$ ). Finally, the jet flows down to a point close to the end of the conical shape, at which the deformation rate reaches the value critical value DST value, and the jet enters the DST regime and exhibits several features of a solid-like structure.

The extension rate inside the cone at the output of the funnel, $\dot{\epsilon}=\frac{\partial}{\partial t} \frac{\delta H}{H}$ where $H$ is the height of a given volume

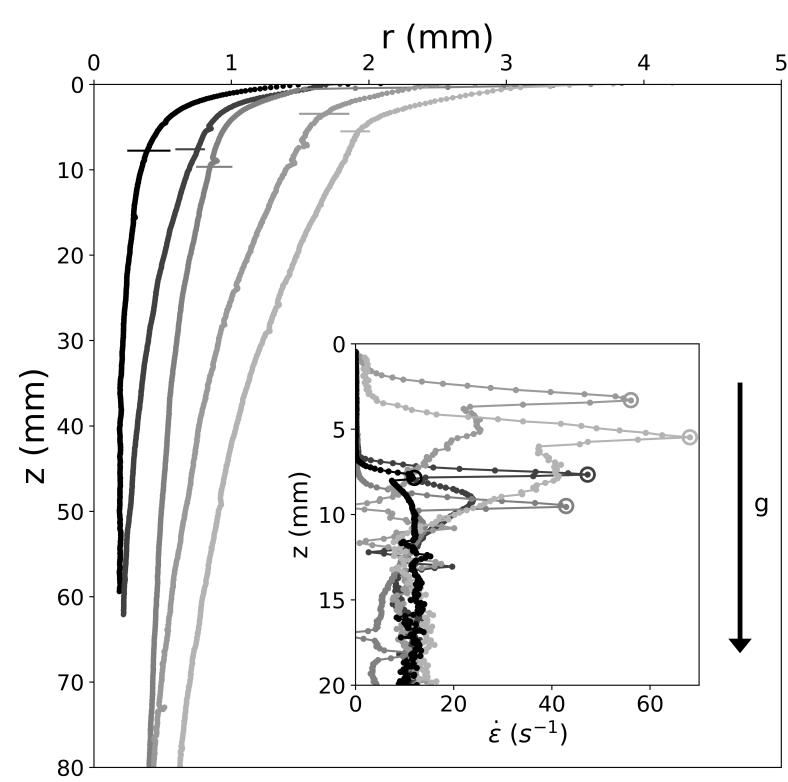

Fig. 4. Shape of the falling jet at volume fraction $\phi=0.5325$ for different output funnel radii, $a$. From black to light grey, $a=1.5,1.75,2,2.5$ and $3.5 \mathrm{~mm}$. Inset : extension rate $\dot{\epsilon}$ along the jet according to eq. 3 . The color code is the same as in the main graph. The position of maximal extension rates (indicated by circles) are indicated by horizontal bars along the jet shape in the main graph.

at the entrance of the cone, immediately at the output of the funnel, and $\delta H=H_{\text {out }}-H_{\text {in }}$ its variation inside the conical shape, can be estimated from the flow rate $(0.17$ $\mathrm{mL} \cdot \mathrm{s}^{-1}$ for $\left.\phi=0.53\right)$ and the shape of the cone $(\epsilon=$ $\left(\frac{R_{\text {in }}}{R_{\text {out }}}\right)^{2}-1=10.1$, where $R_{\text {in }}=1.5 \mathrm{~mm}$ and $R_{\text {out }}=0.45$ $\mathrm{mm}$ ). The volume of the cone being $4.5 \cdot 10^{-2} \mathrm{~mL}$, the time spent in the conical shape is $4.5 \cdot 10^{-2} / 0.17=0.28 \mathrm{~s}$ and the extension rate $\dot{\epsilon}=10.1 / 0.28=36 \mathrm{~s}^{-1}$, of the same order of magnitude than the critical DST rate (Fig. 1), $23.1 \mathrm{~s}^{-1}$. This result contrasts with the result of [15] where extension controlled experiments showed that DST occurs at smaller rates under an extension geometry than in shear geometry, but is consistent with simulation results [16]. The stress at the bottom of the conical section of the jet, below the output of the funnel and above the part with a roughly constant radius, under the weight of the jet may be moreover estimated as $\rho g h_{c y l}=350 \mathrm{~Pa}$ where $h_{c y l}$ is the height of the column underneath, $70 \mathrm{~mm}$ (see Fig. 3 (a)), much above the critical DST stress of suspensions at $0.53 \leq \phi \leq 0.54$ (between 11 and $17 \mathrm{~Pa}$ ).

\section{Discussion}

Let us first consider the time evolution of the mass. The total weight of the funnel is measured (Fig.6). For volume fractions strictly smaller than $\phi=0.53$, the jet flows 


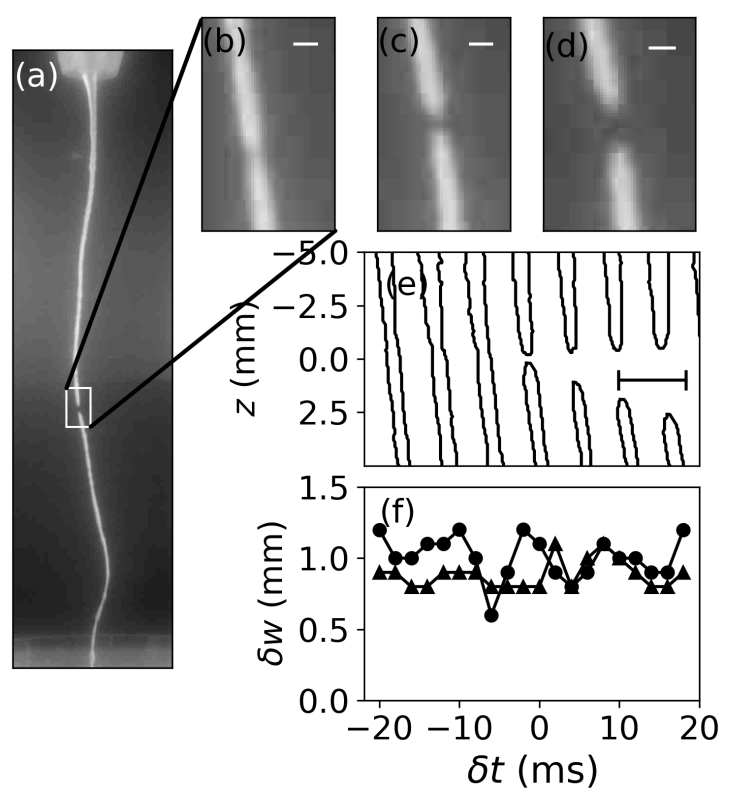

Fig. 5. Detail of the rupture of a jet at $\phi=0.5325$. a) Full image of the jet immediately after a rupture event. (b) to (d) detailed images of the jet at the rupture point taken at $2 \mathrm{~ms}$ intervals. Withe bars correspond to $1 \mathrm{~mm}$. (e) Shape of the jet before and after rupture at $2 \mathrm{~ms}$ intervals. The shapes are translated horizontally for clarity. The length of the horizontal bar is $5 \mathrm{~mm}$. f) Width of the jet above (disks) and below (triangles) the rupture event, in a time interval [-20 ms, $20 \mathrm{~ms}$ ] around a rupture event.

out of the funnel and, when $\phi$ is larger than 0.50 , breaks into droplets (Fig. 3). In this regime, the weight decreases smoothly. Conversely, for volume fractions larger than 0.53 , well-defined steps are observed, separated by regions where the mass is constant. This implies that the weighing device does not measure the mass of the funnel and of the remaining suspension inside the funnel at a given time, but that it also measures the weight of the jet underneath the output. The mass losses correspond to ruptures of the jet, and between two such ruptures, the mass remains constant although the jet continues flowing out of the funnel. As a consequence, tensile stress is transmitted along the jet. This result is consistent with previous observations that, in the DST regime, stress is transmitted by a stress supporting structure, and suggests that, in our geometry, forces propagate up to a distance larger than the diameter of the jet. The surface of the jet is put under tension by normal stresses [17], and strong capillary forces, generated by the menisci at the surface of the jet, transmit tensile stress along the column.

One may assume that DST occurs at the highest sheared regions (Fig. 4), 3 to $10 \mathrm{~mm}$ from the funnel output, where the local extension rate is of the order of the DST shear rate, and then a solid-like rope falls down under the effect of gravity. The lifetime of the DST state depends on the volume fraction of the suspension, but has been measured

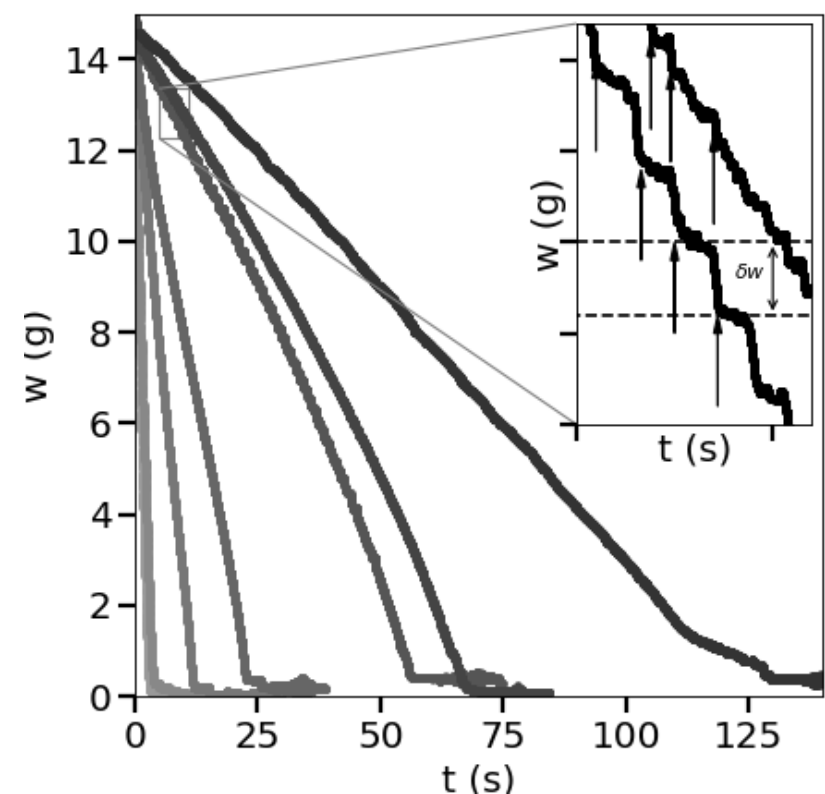

Fig. 6. Evolution of the mass of the funnel as a function of time for $\phi=0.49,0.5,0.52,0.525,0.53,0.535$ and 0.5375 , from left to right. Inset: zoom of the mass loss for $\phi=0.53$ (bottom curve) and 0.535 (top curve). Mass losses $\delta w$ are defined by abrupt losses of mass, as indicated by arrows.

to be of the order of few seconds after cessation of application of shear stress [12], long enough so that the jet stays in the DST state until it breaks. It is fed with particles inside the cone underneath the output of the funnel, and remains attached to the funnel by capillary tension of the cone. Then, the second part of the jet shape whose radius slightly decays with height transmits stress, both by the fabric of force chains in volume and by capillary forces at the surface. At some point, the jet exhibits brittle fracture, as observed in [17]. The fracture events are very different from breaking of liquid columns, that involves thinning of the column before rupture [23]. Here (Fig. 5), solid-like fracture is observed, during which the diameter of the jet remains constant close to the fracture event. These fractures lead to steps in the evolution of mass with time. These steps are well defined and may be easily identified for $\phi=0.53$ but their amplitude becomes of the same order of magnitude of the horizontal force exerted by the oscillating jet on the funnel, when the volume fraction increases (Fig. 6(a) inset) and they cannot unambiguously be distinguished from noise of the weight curve, that is mainly induced by the forces exerted by the jammed structure inside the cone underneath the funnel output, onto the funnel itself.

Finally, in this regime, the jet is no longer vertical, but transverse oscillations propagate along the jet. We observe this instability for output diameters ranging from 3 to 7 $\mathrm{mm}$. At $\phi=0.52$, the jet remains stable whereas at larger output diameters, the increase of the deformation rate at the output of the funnel leads to jet instability. Their ve- 


\section{Conclusion}

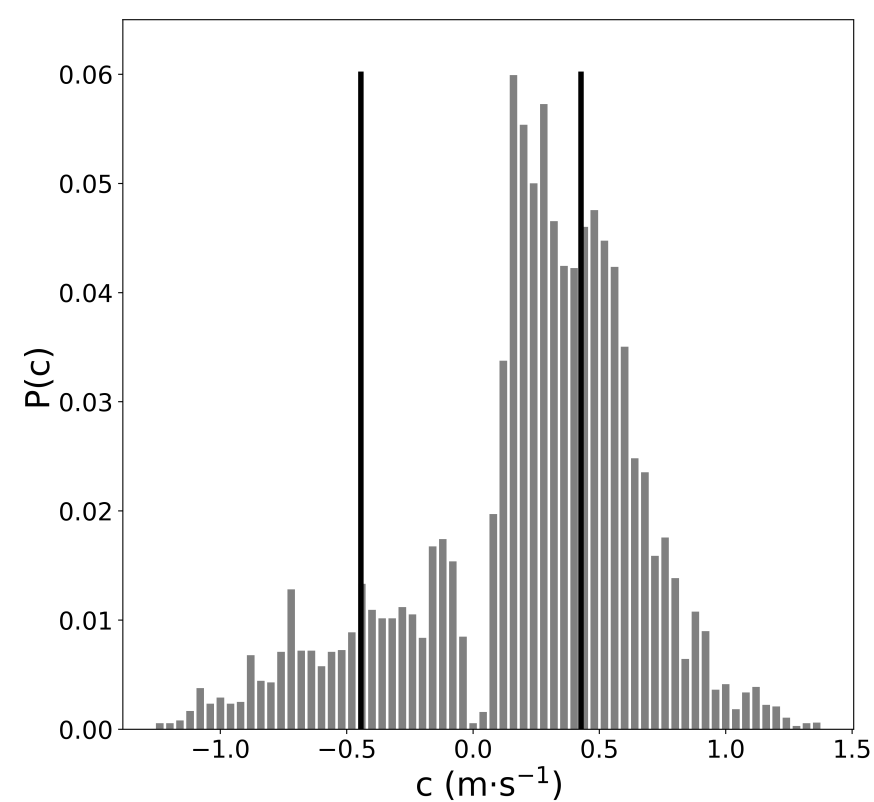

Fig. 7. Probability densities of transverse waves celerities for $\phi=0.5375$ ((b)). Positive values correspond to waves propagating downwards and negative values to waves propagating upwards. Thick black lines are average velocities of waves travelling downward and upward.

locity is measured by the evolution of their maxima as a function of time. Some waves travel upwards whereas some travel downwards. The distribution of velocities is given in Fig. 7 for $\phi=0.5375$. Waves travelling downward (positive velocities) and upward are measured, but most of them $(77 \%)$ travel downward. This suggests that the breaking of chunks at the bottom of the jet is not the dominant mechanism of wave generation. Most of them seem to be generated in the conical region at the output of the funnel, when heterogeneities of the concentration or of the deformation rate induce the formation of chunks of particles. The generation of these oscillations may also be due to stress fluctuations $[25,30]$ in the DST regime, at any position along the column. Moreover, the average celerity of waves travelling upward is $0.43 \mathrm{~m} \cdot \mathrm{s}^{-1}$ and downward $-0.44 \mathrm{~m} \cdot \mathrm{s}^{-1}$. Surprisingly, they do not seem to be advected by the falling jet whose average velocity of 0.16 $\mathrm{m} \cdot \mathrm{s}^{-1}$ would lead to Doppler shift of travelling waves.

Let us model the jet as a rope under its own weight. The propagation of a single wave along the rope would have the velocity $c=\sqrt{\ell g}=1 \mathrm{~m} \cdot \mathrm{s}^{-1}$ where $\ell$ is the length of the jet. This value is twice as large than the average measured phase velocity. This is due to the fact that we observe superpositions of downward and upward travelling waves. Indeed, depending on the phase shift between the two, the apparent phase velocity will change. Assuming a reflection coefficient equal to 0.5 , the average phase velocity is equal to $0.5 \mathrm{~m} \cdot \mathrm{s}^{-1}$, in agreement with measurements.
Our observation of a falling jet of a concentrated suspension confirms that discontinuous shear thickening is observed in jet flow geometries and occurs at extension rates similar to shear rates at the DST transition. Associated with strong confinement stress induced by capillary stresses at the surface, this allows the jet to sustain tensile stress and to propagate transverse waves.

\section{Author contribution statement}

$\mathrm{PH}$ conceived the study. ML, AS and JS carried out the experiments. ML, DL, and $\mathrm{PH}$ prepared the figures and drafted the manuscript. All authors discussed and interpreted the results, edited the text, and finalized the manuscript.

\section{References}

1. E. Brown, N.A. Forman, C.S. Orellana, H. Zhang, B.W. Maynor, D.E. Betts, J.M. DeSimone and H.M. Jaeger. Nature Materials, 9, 220 (2010).

2. E. Brown and H. M. Jaeger. Reports on Progress in Physics, 77, 046602 (2014).

3. B. M. Guy, M. Hermes, and W.C.K. Poon. Physical Review Letters, 115, 088304 (2015).

4. H.A. Barnes. Journal of Rheology, 33, 329 (1989).

5. L.C. Hsiao, S. Jamali, E. Glynos, P.F. Green, R.G. Larson, and M.J. Solomon. Physical Review Letters, 119, 158001 (2017).

6. D. Lootens, H. van Damme, Y. Hémar, and P. Hébraud. Physical Review Letters, 95, 268302 (2005).

7. N. James, E. Han, J. Jureller, and H. Jaeger. Nature Materials, 17, 965 (2018).

8. M.M. Denn, J.F. Morris, and D. Bonn. Soft Matter, 14, 70 (2018).

9. R. Mari, R. Seto, J.F. Morris, and M.M. Denn. Journal of Rheology, 58, 1693 (2014).

10. X. Cheng, J.H. McCoy, J.N. Israelachvili, and I. Cohen. Science, 333, 1276 (2011).

11. I.R. Peters, S. Majumdar, and H.M. Jaeger. Nature, 532, 214 (2016).

12. F. Ianni, D. Lasne, R. Sarcia and P. Hébraud. Physical Review E, 74, 011401 (2006).

13. M. Wyart and M.E. Cates. Physical Review Letters, 112, 098302 (2014).

14. M. E. Cates, J. P. Wittmer, J.-P. Bouchaud, and P. Claudin. Physical Review Letters, 81, 1841 (1998).

15. E.E. Bischoff White, M. Chellamuthu, and J.P. Rothstein. Rheologica Acta, 49, 119 (2009).

16. R. Seto, G.G. Giusteri and A. Martiniello. Journal of Fluid Mechanics, 825, R3 (2017).

17. M.I. Smith, R. Besseling, M.E. Cates, and V. Bertola. Nature Communications, 1, 114 (2010).

18. E. Han, I.R. Peters, and H.M. Jaeger. Nature Communications, 7, 12243 (2016).

19. S.R. Waitukaitis and H.M. Jaeger. Nature, 487, 205 (2012). 
20. P. Coussot. Mudflow Rheology and Dynamics. CRC Press (1997).

21. H.M. Laun, R. Bung, and F. Schmidt. Journal of Rheology, 35, 999 (1991).

22. M. D. Haw. Physical Review Letters, 92, 185506 (2004).

23. J. Eggers and E. Villermaux. Reports on Progress in Physics, 71, 036601 (2008).

24. J. Ferrand, L. Favreau, S. Joubaud, and E. Freyssingeas Physical Review Letters, 117, 248002 (2016).

25. D. Lootens, H.Van Damme, and P. Hébraud. Physical Review Letters, 90, 178301 (2003).

26. C-P. Hsu, S.N. Ramakrishna, M. Zanini, N.D. Spencer, and L. Isa. Proceedings of the National Academy of Sciences, 115, 5117 (2018).

27. C. Weber Journal of Applied Mathematics and Mechanics, 11, 136 (1931).

28. A.M. Sterling and C.A. Sleicher. Journal of Fluid Mechanics, 68, 477 (1975).

29. R.P. Grant and S. Middleman AIChE Journal, 12, 669 (1966).

30. V. Rathee, D.L. Blair and J.S. Urbach P.N.A.S., 114, 33, 8740-8745 (2017) 